\title{
Adsorption process for phospholipids of different chain lengths at a fluorocarbon/water interface studied by Du Noüy ring and spinning drop
}

\author{
Kirsten Ullmann ${ }^{1} \cdot$ Lukas Poggemann $^{2} \cdot$ Hermann Nirschl ${ }^{1} \cdot$ Gero Leneweit $^{3,4}$ \\ Received: 9 August 2019 /Revised: 22 January 2020 / Accepted: 12 February 2020 / Published online: 6 March 2020 \\ (C) The Author(s) 2020
}

\begin{abstract}
Fluorocarbons are novel systems in the fast-growing fields of diverse biomedical applications and fluorocarbon-water emulsions. However, characterization of these systems with modern measuring techniques such as drop profile analysis tensiometry is almost impossible because of practically identical refractive indexes and high-density differences. Due to the material properties of the fluorocarbon-water system, the invasive Du Noüy ring is the most appropriate method to measure interfacial tensions over long times. However, the influence of the ring on a fluorocarbon/water interface packed with phospholipids needs careful analysis. For the proof of methodology, the spinning drop tensiometry was used for comparison as a non-invasive technique to measure interfacial tension between water and perfluoroperhydrophenanthrene (PFPH) covered by 1,2-dipalmitoyl-sn-glycero-3-phosphatidylcholine (DPPC) proving almost identical results. This demonstrates the validity of the invasive measurement technique for the studied system. The Du Noüy ring method was applied for further measurements of phospholipids with different chain lengths (1,2-dmyristoyl-sn-glycero-3phostphatidylcholine, DMPC; 1,2-distearoyl-sn-glycero-3-phosphatidylcholine, DSPC) which revealed a difference in interfacial adsorption kinetics and equilibrium tensions. The Du Noüy ring tensiometry is appropriate to examine the slow adsorption kinetics of phospholipids emulsifying fluorocarbons. The results enable functional optimization of fluorocarbon emulsions regarding physical emulsification parameters and the selection of lipids.
\end{abstract}

Keywords Interfacial tension · Phospholipids · Fluorocarbon · Adsorption · Du Noüy ring · Spinning drop

Electronic supplementary material The online version of this article (https://doi.org/10.1007/s00396-020-04618-3 ) contains supplementary material, which is available to authorized users.

Kirsten Ullmann

kirsten.ullmann@kit.edu

$1 \quad$ Institute of Mechanical Process Engineering and Mechanics, Process Machines, Karlsruhe Institute of Technology (KIT), Straße am Forum 8, 76131 Karlsruhe, Germany

2 Institute of Mechanical Process Engineering and Mechanics, Gas Particle Systems, Karlsruhe Institute of Technology (KIT), Straße am Forum 8, 76131 Karlsruhe, Germany

3 Association for the Promotion of Cancer Therapy, Carl Gustav Carus-Institute, 75223 Niefern-Oeschelbronn, Germany

4 Abnoba GmbH, Hohenzollernstr. 16, 75177 Pforzheim, Germany

\section{Introduction}

Phospholipids (PLs) are widely used in nutrients and pharmaceutical applications because of their natural occurrence in cell membranes. As an endogenous substance, they are safe to be applied in the food and pharma industry and often utilized as natural emulsifiers [1]. Understanding the adsorption kinetics of PLs helps to improve nanoemulsions. From the thermodynamic behaviour of interfacial layers, the optimum area per lipid molecule is derived and can be adduced to determine the PL concentration for the emulsifying process as well as give information about the emulsion stability [2]. Thus, studies of interfacial tensions between PL and an oil or organic phase are of high interest to understand the adsorption kinetics and improve the emulsification process. Organic phase-water or water-air interfaces and the adsorption behaviour of PL used in these systems have been described in a variety of literature [3-5]. However, not many experimental data exist about 
interfacial tensions with the organic phase being a fluorocarbon including PL as surfactants. Commonly used oils show a high miscibility with PL and, hence, favour the formation of gel phases at interfaces $[6,7]$. In addition, emulsions from natural oils are less stable and unsaturated oils tend to quickly oxidize [2]. Fluorocarbons have been found to be good for the use of pharmaceutical emulsions. Most fluorocarbons are inert, very stable, biocompatible and a possible alternative in biomedical use [8-10]. The application of fluorocarbon and perfluorocarbon nanoemulsions expanded over the last couple of years. They are utilized for magnetic resonance imaging (MRI) as a contrast agent $\left({ }^{19} \mathrm{~F}\right)$ to detect tissue artefacts such as venous thrombosis at an early stage [11] and various other applications in the field of MRI [12,13]. Moreover, Chattaraj et al. [14] added PLs in various compositions to enhance the contrast and examine lipid layer behaviour. Fluor-containing compositions of drug or protein delivery systems were examined by Zhang et al. [15], who took advantage of the fluorination effect of fluoroamphiphiles for a more efficient protein delivery. An additional imaging mode is the positron emission tomography (PET). Amongst the required contrast agents are fluorocarbons $\left({ }^{18} \mathrm{~F}\right)[16,17]$. To summarize, fluorocarbons are inert, very stable, and biocompatible excipients in pharmaceutical emulsions. Moreover, they offer the advantage of being a contrast agent as natural or synthetic isotope for MRI and PET. They are ideal oxygen suppliers for organ transports because of their high gas solubility and volatile fluorocarbons that enable triggered release of APIs because of the low evaporation temperature [18].

All these applications make use of nanoemulsions which is why their characterization comes to the fore. Phospholipids as natural emulsifiers influence the nanoemulsions and their interfacial characteristics. However, only a few data on interfacial tensions of fluorocarbon oils have been published so far [19].

Interfacial tensions are commonly measured with optical methods. Profile analysis tensiometry (PAT) has been used extensively to determine the adsorption process of surfactants [20-22] and is considered as non-invasive. The Du Noüy ring is one of the first established methods that allowed interfacial measurements [23]. It is a non-optical measurement especially used for fast measurements of pure liquid surface tensions [24]. The ability to quickly obtain measurement data of an unknown system, however, is associated with the disadvantage that artefacts can occur with the ring method [25]. With ongoing research, more methods were introduced [26].

Standard measuring techniques such as PAT were tested but were found to be not suitable for our examined system because the large density difference between both phases, which lead to a rapid drop detachment, impede the formation of long-term stable drops needed for PAT. Additionally, the camera cannot detect the droplet automatically because of almost identical refractive indices $n$ of perfluoroperhydrophenanthrene (PFPH) and phosphate buffer $(\Delta n=0.0004)$. However, automatic image detection is crucial for this specific non-invasive technique to determine the adsorption process. To influence the refractive index of the aqueous phase and allow automatic image detection with PAT, a saccharose and sodium chloride solution were studied. With the dispersed PL in the aqueous phase measurements were performed for $2000 \mathrm{~s}$. However, equilibrium interfacial tension was not reached within this time. In addition, the performed experiments showed high standard deviations and no change in interfacial tensions depending on the concentration of PL (data not shown). It was concluded that standard measuring techniques are not suitable to detect interfacial tensions between PFPH and a PL suspension. This is why the Du Noüy ring method was chosen here as it allows to measure immiscible phase of high-density differences irrespective of their refractive indexes and the turbidity of an aqueous PL suspension. The Du Noüy ring method is generally seen as prone to error and, thus, validated with a second measurement technique. Similar to our approach, Kovach et al. studied Janus emulsions of silicon oil, olive oil, water, phospholipids and other emulsifiers, but a rigorous comparison of the du Noüy and the spinning drop method has not been provided so far [27]. The spinning drop tensiometer was used for comparison because it provides exact data, especially in the range of lower interfacial tensions [28] and is based on a completely independent physical principle. However, experiments performed with the spinning drop take much longer for our system as for typical materials studied with it, as equilibrium state for concentrationdependent interfacial tensions has to be reached under rotation. Additionally, it is usually applied for systems with very small interfacial tensions which do not apply to our system. In contrast to that, the Du Noüy ring method allows several measurements simultaneously which enables to examine a greater amount of different concentrations and PL in a shorter period of time due to parallel sample equilibration. However, only few studies show phospholipids as natural surfactants and their adsorption kinetics at a water-oil interface measured with the $\mathrm{Du}$ Noüy ring such as Benmekhbi et al., who investigated the adsorption kinetics of Span80 at a liquid/liquid interface [29].

The aim of this work is to show that the Du Noüy ring is an appropriate method to measure interfacial tensions of systems, which are impossible to characterize with other modern techniques such as PAT. Furthermore, our validation of the Du Noüy ring method can set the foundation for large-scale screenings of fluorocarbon or other unknown systems with samples being prepared in parallel for longterm adsorption outside the measurement device and a very quick measurement process taking only a few minutes per sample. Additionally, we applied the method presenting different interfacial measurements of PL suspensions with the $\mathrm{PL}$ of various chain lengths and, for the first time, the fluorocarbon oil perfluoroperhydrophenanthrene (PFPH). The selected phospholipids have the same phosphatidylcholine 
head group in common but vary in their chain length from 14 to 18 carbons.

\section{Material and methods}

\section{Materials}

Lipoid (Ludwigshafen, Germany) provided all phospholipids used for the experiments. The synthetic phospholipids 1,2dimyristoyl-sn-glycero-3-phostphatidylcholine (DMPC, (fatty acid chain lengths/double bonds =14:0), 1,2-dipalmitoylsn-glycero-3-phosphatidylcholine (DPPC, 16:0) and 1,2distearoyl-sn-glycero-3-phosphatidylcholine (DSPC, 18:0) were received in powder form and dissolved in ethanol (99\%, Carl Roth GmbH, Karlsruhe, Germany) for preparation of stock solutions. Cholesterol (chol.) was purchased from Carl Roth ( Karls r u h e, G e r m a n y ). Perfluoroperhydrophenanthrene (PFPH) was purchased from F2 Chemicals (Preston, UK).

\section{Preparation of lipid suspensions}

For all experiments, stock suspensions of $20 \mathrm{mM}$ of either pure lipids were prepared. To prepare the stock suspensions, the film hydration method was applied, and phospholipids were dissolved in $99 \%$ ethanol. The film was dried in a rotary evaporator (Heidolph Instruments, Schwabach, Germany) and rehydrated in double-distilled water (ddH2O, Arium Pro, Sartorius, Göttingen, Germany). [30] Because of a very low solubility of phospholipids in PFPH $(<10-6 \mathrm{mM})$ stock solutions were prepared with ddH2O. For better dissolution of the lipids, ultrasonic sound with a $100 \%$ cycle and $10 \%$ amplitude (with sonotrode Digital Sonifier 450, Branson Ultrasonic Corporation, Danbury, USA) was applied for $10 \mathrm{~s}$ followed by a $50 \%$ cycle and $10 \%$ amplitude for $10 \mathrm{~min}$. For longer stability, all stock solutions were extruded (LIPEX $^{\mathrm{TM}}$ Extruder, Transferra Nanosciences, Burnbary, CA) through 400- and 200-nm-ion beam track-etched membranes (Whatman, GE Healthcare, Little Chalfont, UK) 20 times each. Both applications led to a suspension of vesicles ranging from 100 to $200 \mathrm{~nm}$ which was verified by dynamic light scattering (Zetasizer Nano ZSP, Malvern Instruments, Worcestershire, UK). All stock suspensions were stored in the fridge at $4{ }^{\circ} \mathrm{C}$. The final phospholipid concentration after extrusion and ultrasonic sound was determined by measuring the amount of phosphate colorimetrically according to Fiske [31]. Prior using the stock solution, it was brought to room temperature $\left(20{ }^{\circ} \mathrm{C}\right)$ and diluted to the desired concentration.

\section{Tensiometry}

Interfacial tension was measured with the Du Noüy ring tensiometer DCAT EC11 (DataPhysics, Filderstadt, Germany) (compare schematic illustration in Fig. 1 left) which is placed in a temperature controlled room $\left(20^{\circ} \mathrm{C}\right)$ and run at $20.0 \pm$ $0.1^{\circ} \mathrm{C}$ using a thermobarrel and a water bath. The accuracy of the platinum-iridium ring (DataPhysics, Filderstadt, Germany) was validated by determining the surface tension of water ( $72 \mathrm{mN} / \mathrm{m}$ [32]). Additionally, the interfacial tension between water and perfluoroperhydrophenanthrene $(52.4 \mathrm{mN} /$ $\mathrm{m}$ ) was measured and used as the initial interfacial tension for all measurements. The interfacial tension was determined for three different PL: DMPC, DPPC and DSPC. For these measurements, $15 \mathrm{~mL}$ of the oil phase was transferred in the glass vessel which was cleaned with isopropanol and dried in a heat chamber. To avoid mixing of the oil and water phase, the aqueous phase containing PLs was carefully pipetted along the glass wall on top of the oil phase $(15 \mathrm{~mL})$. To receive the concentration-dependent interfacial tension of the examined PLs, the aqueous phase was concentrated. The increase of concentration was performed by adding the necessary amount of PL stock solution stepwise to the aqueous phase of the same glass vessel. Interfacial tension was measured directly after increasing the concentration as well as after reaching the quasi-equilibrium state. The equilibration time for each phospholipid was determined for different concentrations prior starting with the test series and used for all further experiments. Each experiment was carried out in triplicates. Within each experiment, measurements were performed three times with less than $1 \mathrm{~min}$ in between, before increasing the concentration. Flame treatment after each measurements cleaned the ring.

The Du Noüy ring method was validated with the spinning drop tensiometer (Spinning Drop Video Tensiometer SVT20, DataPhysics, Filderstadt, Germany, Fig. 1, right). Because of similar refractive indices of the examined systems (phospholipid suspension $R I=1.331, R I_{\mathrm{PFPH}}=1.335$ ), the circumference of the forming droplet cannot be identified via the supporting camera system but with a manually applied circumference. To ensure an equally correct measurement without automatic image acquisition, validation experiments with water and hexanol were performed.

The validation was performed with the interfacial measurement of DPPC only. Both phases were kept at $20.0 \pm 0.1{ }^{\circ} \mathrm{C}$ at all times. The oil phase was filled in the capillary, ensuring it is free of any gas bubbles. The aqueous phase with DPPC was diluted depending on the examined concentration and added with a syringe that is stung through the septum, while the capillary was brought into rotation with $\omega=500 \mathrm{rpm}$ to avoid the droplet to attach to the glass wall. With an increasing angular velocity $\omega$, the droplet reaches a cylindrical form and used to calculate the radius $r$ by applying the manually 


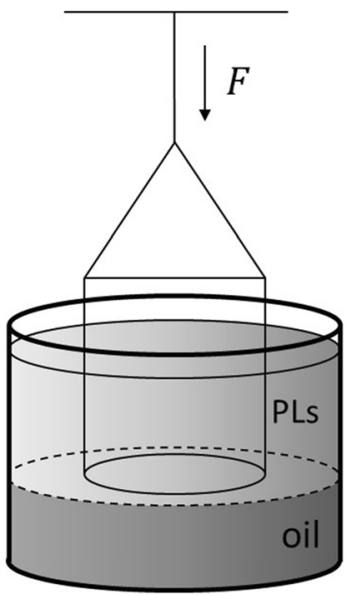

Fig. 1 Schematic experimental set-up of the two measurement methods. Left: The Du Noüy ring measurement. Because of a heavier oil phase, the aqueous phase (PLs) is pipetted on top and the lamella forms by pushing the ring in the oil phase. A resistance force $F$ is measured and used for calculating the interfacial tension $\gamma$. Right: Spinning drop tensiometer. A

set circumference, which allows to apply Eq. (1) and determine the interfacial tension:

$\gamma=1 / 4 \Delta \rho r^{3} \omega^{2}$

Vonnegut et al. deduced Eq. (1) from the minimum state of energy [28]. In order to apply Eq. (1), the length of the droplet ought to be four times longer than the radius. Additionally, the density $\rho$ of both liquids is needed for a calculation. Repetitions of the interfacial tension were obtained by changing the angular velocity at least five times in $200 \mathrm{rpm}$ steps. Equally to the Du Noüy ring method, the determination of the equilibration time is necessary for the final measurements. Thus, in a first step, the droplet circulated as long as no change in interfacial tension could be detected. The equilibration time was found to be $24 \mathrm{~h}$ and used for all further experiments.

The interfacial concentration $\Gamma$ of the PLs was derived from the Gibbs adsorption isotherm by using experimental data in a $\gamma$ vs $\log c_{0}$ diagram, with $\gamma$ measured at equilibrium state:

$\Gamma=-\frac{c_{0}}{R T}\left(\frac{\partial \gamma}{\partial c_{0}}\right)_{p, T}=-\frac{1}{R T}\left(\frac{\partial \gamma}{\partial \ln c_{0}}\right)_{p, T}$

where $c_{0}$ ist the bulk concentration, $\gamma$ ist the interfacial tension at equilibrium state, $R$ is the gas constant and $T$ is the absolute temperature. The critical micelle concentration (CMC) was then calculated from the intersection of the linear slope $\frac{\partial \gamma}{\partial \operatorname{lnc}_{0}}$ defined by linear regression in the respective concentration range and from the linear fit above the kink at higher concentrations.

\section{Results and discussion}

\section{Equilibration time of DPPC}

To measure interfacial tensions with the Du Noüy ring method, the determination of the equilibration time is essential to carry out further experiments. The setting of the thermodynamic equilibrium and the related adsorption process of PLs is a diffusion controlled process which needs a certain amount of time to evolve. Other measurement techniques such as PAT take advantage of the online measurement, where the interfacial tensions are constantly recorded, and thus the adsorption process. Additionally, it is a non-invasive technique. However, the equilibrium interfacial tension cannot be detected with PAT because the droplet typically falls off after about $10-30 \mathrm{~min}$. Therefore, an extrapolation towards equilibrium is carried out instead of an infinite measurement. The Du Noüy ring is an invasive technique, and online measurements are not possible, but the advantage of the technique is that the longterm interfacial tensions can be detected easily. The adsorption behaviour can equally be monitored over the same period of time. For comparison of results and concentration-dependent measurements, the equilibration time of DPPC, which was the PL chosen for validation experiments, was determined prior to further experiments. Results are shown in Fig. 2a. It depicts the time until no change in interfacial tension between the aqueous DPPC suspension and PFPH is detected. The four tested concentrations of DPPC $(0.1,0.13,0.14$ and $0.29 \mathrm{mM}$ ) show the same results in terms of their equilibration time. Experiments were run for $168 \mathrm{~h}$, but after $48 \mathrm{~h}$, no significant change in the interfacial tension can be observed. The extrapolated equilibrium interfacial tension including a $95 \%$ confidence interval for standard deviation is in 


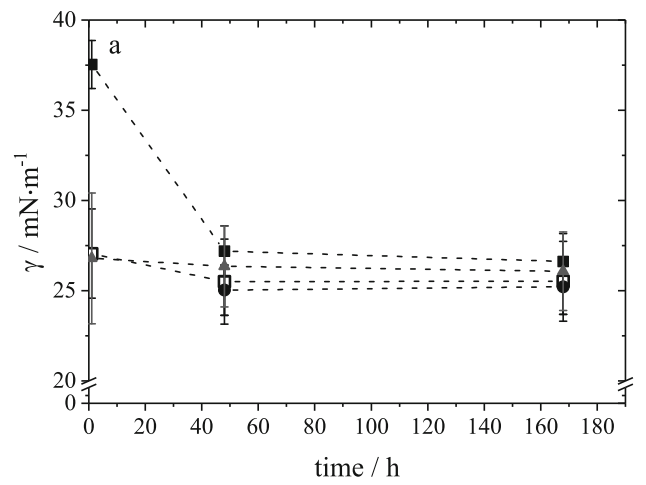

Fig. 2 Equilibration time for the PL DPPC to reach thermodynamic equilibrium when the interfacial tension $\gamma$ is measured with the $\mathrm{Du}$ Noüy ring. a Interfacial tension $\gamma(t)$ displayed vs time $t$. (- - DPPC $0.1 \mathrm{mM},-\square-$ DPPC $0.14 \mathrm{mM},-\bullet-$ DPPC $0.13 \mathrm{mM},-\boldsymbol{\Delta}$ - DPPC

accordance with the measured interfacial tension after $48 \mathrm{~h}$ $\left(\gamma_{0.1 m M}(t \rightarrow \infty)=25.56 \pm 1.64 \mathrm{mN} / \mathrm{m}, \gamma_{0.1 m M}(48 h)=\right.$ $27.18 \pm 1.42 \mathrm{mN} / \mathrm{m}$ and $\gamma_{0.14 \mathrm{mM}}(t \rightarrow \infty)=25.32 \pm 0.91 \mathrm{mN} /$ $\mathrm{m}, \gamma_{0.14 m M}(48 h)=25.50 \pm 2.36 \mathrm{mN} / \mathrm{m}$ ) (Fig. 2b). Thus, further experiments with DPPC were carried out with at least $48 \mathrm{~h}$ before measuring the equilibrium interfacial tension.

\section{Influence of the ring}

In addition to the determination of the equilibration time of the non-disturbed interface, investigations on a possible influence of the ring on the interface were performed. The aim was to test the period of equilibration after contact of the ring with the interfacial monolayer and a possible artefact after too fast repetition as the Du Noüy ring method is an invasive technique. Using the Du Noüy ring, a physical contact disturbs the interface and may thus distort the tension between both fluids. To find out to what extent the physical contact of the ring changes the interfacial tension reversibly between the measurements, the same sample was measured three times consecutively with a measurement period of approx. $10 \mathrm{~min}$ and, additionally, 120 h later (Fig. 3). Furthermore, it was monitored whether the phospholipids adsorb at the interface after the ring is removed.

Blank bars depict the interfacial tension determined at the first measurement. Within one sample, the interfacial tension increases with each consecutive measurement at $48 \mathrm{~h}$. With the repeated immersion and pulling out of the ring at the interface, the adsorbed phospholipids are removed which results in a higher interfacial tension when measuring a second and third time. Yet, $120 \mathrm{~h}$ later (grey bars), the interfacial tension of the same sample decreases again to practically the same value as compared to before which is then stable for the first and second measurement, showing a distorted interface only when measured for the third time. This behaviour particularly appears in the regime of moderate concentrations when the PL adsorb at the interface and unless it is fully covered at

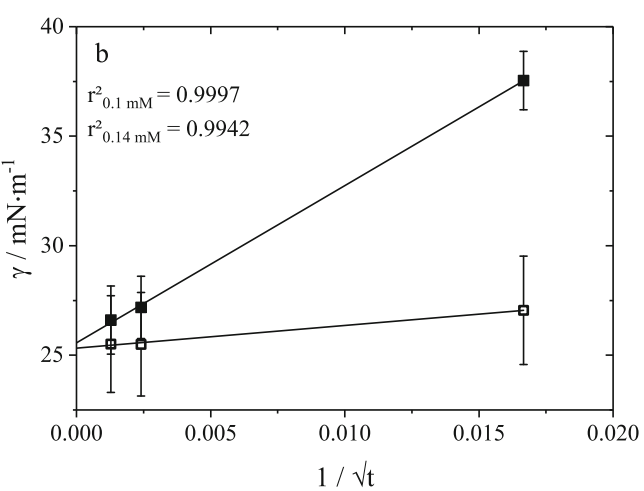

$0.29 \mathrm{mM})$. b Selected data from the left hand side, but displayed as $\gamma(t)$ vs. $1 / t^{1 / 2}$, with $r^{2}$ as the variation coefficient. Standard deviation calculated from $n=3$ experiments. ( - - DPPC $0.1 \mathrm{mM},-\square-$ DPPC $0.14 \mathrm{mM}$, - linear extrapolation)

saturation, indicating a possible stress relaxation process [33]. It also leads to the conclusion that in the adsorption regime of the PL, the first measured value for the interfacial tension is the only accurate due to the invasive distortion of the interfacial layer (Fig. 3, black arrow). While this is true for higher concentrations, very low concentrations of PL are not affected by this phenomenon.

Additionally, a possible influence of the ring was investigated during the adsorption process of the PL. Thus, two independent experimental set-ups were examined (Fig. 3b): Setup 1 consisted of time-resolved measurements, with measurements at the beginning of the experiment as well as in between to monitor the development. Set-up 2 was measured in the beginning and at the end only. The time-resolved measurements of the determined interfacial tension show a decrease of the tension with time. The single measurement at the beginning and at the end only matches these results and reveals a similar value of interfacial tension of $0.072 \mathrm{mM}$ DPPC after $48 \mathrm{~h}$ (Fig. 3; $42 \mathrm{mN} / \mathrm{m}$ ). The discrepancy in interfacial tension of the first measurements of each sample is due to nonequilibrium conditions. Two independent sample set-ups were measured at almost equal points in time. Limited accuracy in determining the adsorption kinetics is due to non-equilibrium conditions of the monolayer itself, but as shown in Fig. 3b, the equilibrium interfacial tension is measurable at high accuracy. These results indicate that even with repeated physical contact between the ring and the interface, the adsorption process of phospholipids is not disturbed irreversibly over a longer period of time.

\section{Method comparison}

Based on the determined equilibration time of DPPC, the Du Noüy ring method was used to determine the concentrationdependent interfacial tension. Some of the previously stated assumed difficulties such as a potential destructive influence of the ring could already be proved not to influence the first 


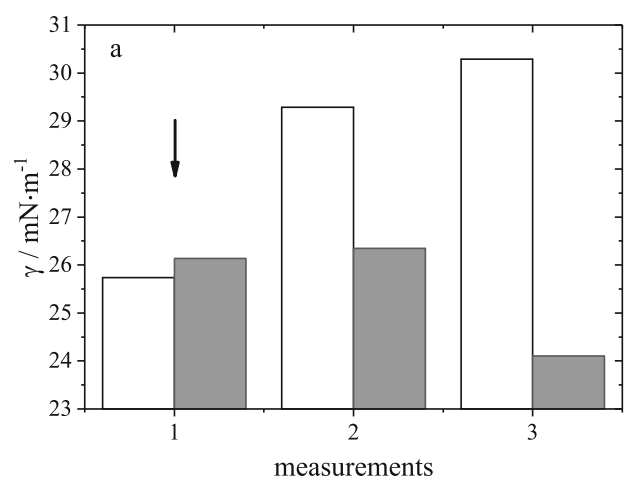

Fig. 3 a Determination of equilibration time until the system is regenerated and equilibrium state is reached after the physical contact with the Du Noüy ring. Exemplary increase of interfacial tension between three measurements of the same sample of $0.14 \mathrm{mM}$ DPPC. Blank bars ( $\square$ ) show the measurement after $48 \mathrm{~h}$, performed three times consecutively (measurement 1,2 and 3 , each taking approx. 3 min, $10 \mathrm{~min}$ in total), grey bars $(\square)$ depict the same sample but measured after $168 \mathrm{~h}$ total and again three times in a row. The arrow indicates that

measurement in a time series of several days, while consecutive measurements within less than an hour indeed show a deteriorating effect. The accuracy of single measurements of

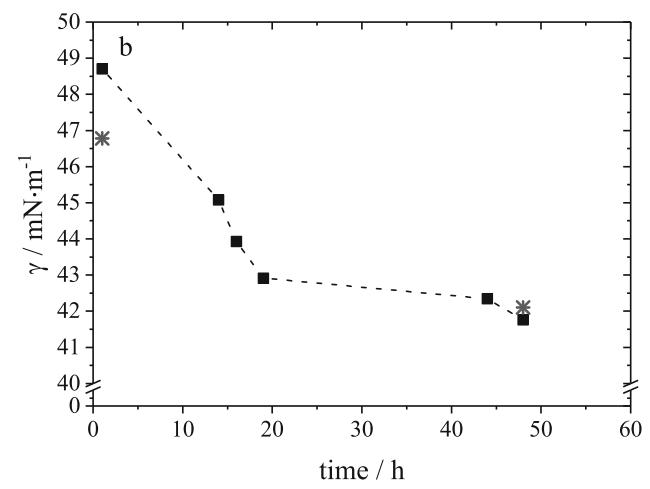

the first measurement point is used for the concentration dependent curve. b Proof that the repeated contact with the Du Noüy ring does not influence the final equilibrium interfacial tension by means of two independent samples of $0.072 \mathrm{mM}$ DPPC. Evolution of the interfacial tension of $0.072 \mathrm{mM}$ DPPC with time (- - DPPC time resolved) and the influence of the ring when it physically touches the interface (* DPPC beginning and end)

the Du Noüy method was demonstrated in comparison to the spinning drop tensiometer as a non-invasive technique (Fig. 4a). In addition, the interfacial tension as a function of
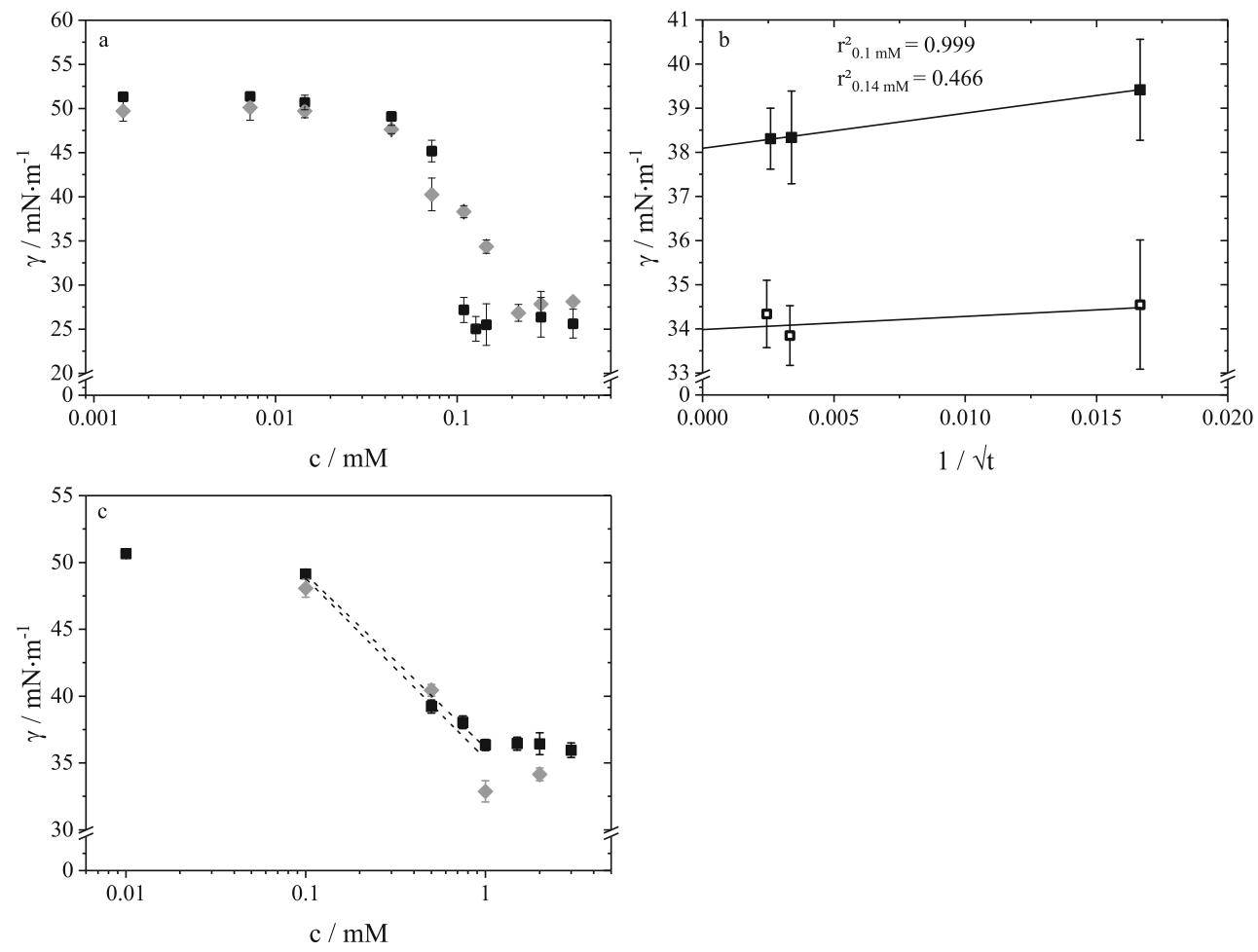

Fig. 4 a Comparison of the accuracy of the Du Noüy ring method to the non-invasive spinning drop tensiometer to determine the interfacial tension $\gamma$ of different DPPC concentrations $c$ ( measurement with Du Noüy ring, $\gg$ measurement with the spinning drop). b Two different concentrations of DPPC measured with the spinning drop tensiometer and displayed as $\gamma(t)$ vs. $1 / t^{1 / 2}$, with $r^{2}$ as the variation coefficient. Standard

deviation was calculated from $n=3$ experiments ( DPPC $_{\mathrm{SD}} 0.1 \mathrm{mM}$, $\square$ $\mathrm{DPPC}_{\mathrm{SD}} 0.14 \mathrm{mM}$ - linear extrapolation). c Comparison of the accuracy of the Du Noüy ring method to the non-invasive spinning drop tensiometer to determine the interfacial tension $\gamma$ of different E80 concentrations $c$, a PL that does not form phase transition ( $\square$ measurement with Du Noüy ring, $\diamond$ measurement with the spinning drop) 
time for two concentrations of DPPC measured with the spinning drop is depicted (Fig. 4b). The extrapolated equilibrium interfacial tensions gained from the $\gamma(\mathrm{t})$ vs. $t^{-1 / 2}$ diagram reveals that it is within the standard deviation of the measured interfacial tension after $24 \mathrm{~h}$. The comparison of spinning drop results for $c=0.1 \mathrm{mM}$ shows $\gamma(t \rightarrow \infty)=38.09 \pm 0.25 \mathrm{mN} / \mathrm{m}$, $\gamma(24 h)=38.34 \pm 1.1 \mathrm{mN} / \mathrm{m}$; and for $c=0.14 \mathrm{mM}, \gamma(t \rightarrow$ $\infty)=33.98 \pm 0.35 \mathrm{mN} / \mathrm{m}, \gamma(24 h)=33.85 \pm 0.68 \mathrm{mN} / \mathrm{m})$. This proves that equilibrium is reached within an error margin smaller than the standard deviation after a surface age of $24 \mathrm{~h}$.

Figure 4 shows that both methods are in good accordance, especially in the lower and upper concentration range when homogeneous suspensions and saturated monolayers exist. Lowest interfacial tension measured with the Du Noüy ring is $25.03 \mathrm{mN} /$ $\mathrm{m}$; the spinning drop method provided $26.83 \mathrm{mN} / \mathrm{m}$ as lowest interfacial tension. The CMC for the spinning drop is $0.258 \mathrm{mM}$ and $0.137 \mathrm{mM}$ for the Du Noüy ring. We assume this discrepancy to come from the dynamic experimental set-up including the influence of shear when measured with the spinning drop in comparison to the static conditions without convection when measured with the Du Noüy ring. The difference between the Du Noüy and the spinning drop method in the intermediate concentration range exactly reflects phase transitions of the DPPC monolayer. For DDPC, a continuous liquid monolayer phase exists for $\gamma \geq 40 \mathrm{mN} / \mathrm{m}$, a liquid and a solid phase coexist for $40 \geq \gamma \geq 27 \mathrm{mN} / \mathrm{m}$ and a solid homogeneous phase for $\gamma<$ $27 \mathrm{mN} / \mathrm{m}$ as described by Vranceanu et al. [34]. Visualizations of the coexistence of solid and liquid phases by fluorescence microscopy are provided by Worthman et al. and Stottrup and Keller $[35,36]$. From these results regarding the phase transitions and their visualizations, the following conclusions can be drawn: (1) The completely static, diffusion-controlled conditions of the fluorocarbon/water interface in the Du Noüy method favour the formation the continuous phase consisting of solids, embedding the fluid phase in the two-phase coexistence. (2) In contrast, the very dynamic, convection-controlled conditions of the spinning drop method lead to a continuous phase consisting of a liquid DPPC monolayer, embedding the DPPC solids in the coexistent range.

This conclusion is supported by taking into account the comparison of the Du Noüy and the spinning drop method for another lipid. For this purpose, the natural phospholipid blend of egg lecithin was chosen, containing $80 \%$ of phosphatidylcholine, termed E80, which contains a variety of saturated and unsaturated PLs of different fatty acid chain lengths. The advantage of this natural blend is that it does not form phase transitions and two-phase coexistent regions. The results show a very high accordance of both methods. This proves the validity of the results of the $\mathrm{Du}$ Noüy ring tensiometer, verified by the spinning drop tensiometer. The standard deviation of both methods are similar on average $\left(\bar{s}_{\text {ring }}=1.162 \mathrm{mN} / \mathrm{m}, \bar{s}_{S D}=0.955 \mathrm{mN} / \mathrm{m}\right)$ indicating that both methods measure the interfacial tension with equal precision.

However, in order to gain one data point measured with the spinning drop consisting of three samples, the measurement device is blocked for $3 \times 24 \mathrm{~h}$ to obtain the data set. In contrast, it only takes around $3 \times 10 \mathrm{~min}$ for three samples to be measured with the Du Noüy ring. Additionally, the capillary of the spinning drop needs to be cleaned in between. In conclusion, the working time required for the spinning drop concentration-dependent graph is at least two times higher, while the 3 repetitive measurements with the Du Noüy ring can all be done in parallel as the beakers with the interfaces can be prepared and stored outside the measurement device and can be exchanged, only needing temperature equilibration of a minute.

Because of the accordance of interfacial tensions and its higher time efficiency the Du Noüy ring method, it was applied for further measurements. As the comparison was further performed with a PL mixture (E80 from egg) and showed similar results, it indicates that both, natural mixtures consisting of different PL head groups and fatty acids as well as pure PL, can be equally measured with the Du Noüy ring.

\section{Equilibration time of PLs of different chain lengths}

After validating the Du Noüy ring method with the spinning drop tensiometry using the PL DPPC, further interfacial tensions of two pure phospholipids, DMPC and DSPC, were examined. As already stated in 3.1.1, the equilibration time is crucial for the experiments and was studied prior to the concentration-dependent measurements, shown in Fig. 5.

The graphs show that the equilibration time changes depending on the phospholipid's fatty acid chain length (Fig. 5).

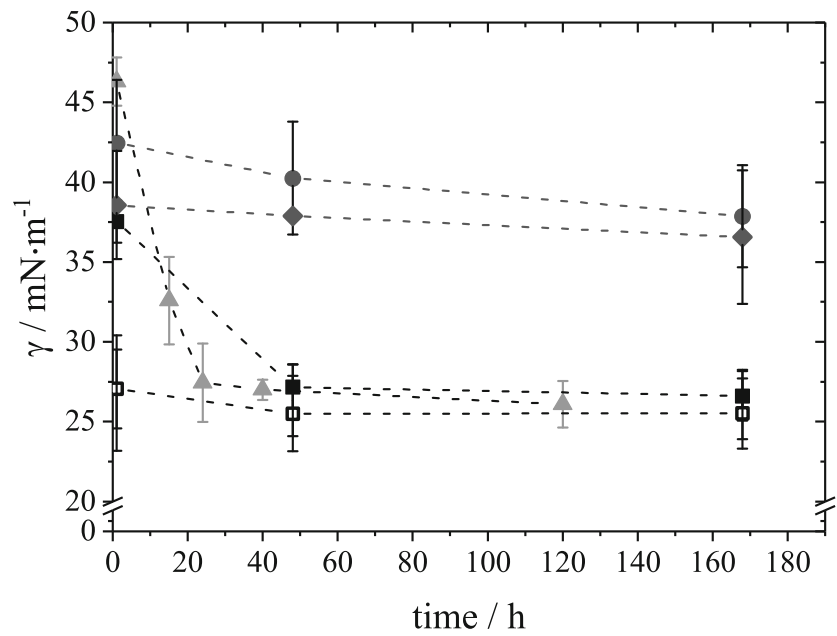

Fig. 5 Change of interfacial tension $\gamma(t)$ with time of the PLs DMPC, $\mathrm{DPPC}$, and DSPC to determine the equilibration time. Standard deviation calculated from $n=3$ experiments. $(-\bullet$ DMPC $0.1 \mathrm{mM},-\square-$ DPPC $0.1 \mathrm{mM},-\square-$ DPPC $0.14 \mathrm{mM},-\triangle-$ DSPC $0.1 \mathrm{mM}, \rightarrow-$ DSPC $0.14 \mathrm{mM})$ 
For $c=0.1 \mathrm{mM}$, DMPC exhibits only little change already between 24 and $48 \mathrm{~h}$. DPPC shows almost no change in interfacial tension between 48 and $168 \mathrm{~h}$ whereas DSPC needs $168 \mathrm{~h}$ for the adsorption process to be complete. This leads to the conclusion that the chain lengths of the fatty acids influence the adsorption process. According to our results, longer chain lengths (18:0, DSPC) need more time (168 h) to adsorb at the interface whereas shorter chain lengths (14:0, DMPC) reach the equilibrium state much faster. The complete results of Fig. 5 are quantitatively analysed in Table 1 for the two concentrations, $c=0.1$ and $0.14 \mathrm{mM}$, showing the decrease $-\Delta \gamma / \Delta \mathrm{t}$ at $48 \mathrm{~h}$ and $168 \mathrm{~h}$ to select the necessary surface age. Additionally, the equilibrium interfacial tension extrapolated towards infinite time is assessed by a $t^{-1 / 2}$ plot and the averaged standard deviations are shown. The higher standard deviation of DSPC $\left(\bar{s}_{D S P C}=3.68 \mathrm{mN} / \mathrm{m}\right)$ is worth mentioning which indicates a more inhomogeneous adsorption process (Table 1) [37].

These results are in accordance with data reported by Kabalnov et al. [19]. Kabalnov used a fluorocarbon as well, in their case perfluorooctyl bromide, and measured surface and interfacial tensions between the aqueous suspension and the fluorocarbon at $22{ }^{\circ} \mathrm{C}$. They estimated that chain lengths longer than $\mathrm{C}_{12}$ take more than a day to reach equilibrium which coincides with our results and emphasizes the influence of diffusion on the interfacial tension. However, equilibration times longer than $24 \mathrm{~h}$ were not considered. Additionally, sonication was applied by Kabalnov et al. prior to the determination of the interfacial tension which accelerated the equilibration at the interface and thus, may not be the actual equilibrium interfacial tension of the PLs.

\section{Interfacial tensions of PLs of different chain lengths}

With the known equilibration time, the interfacial tension and the adsorption behaviour of the examined PLs DMPC, DPPC, and DSPC at different concentrations were determined (Fig. 6). Each data point was derived after the specific equilibration time and a stepwise increase of the concentration. Results of DPPC are shown for comparison.

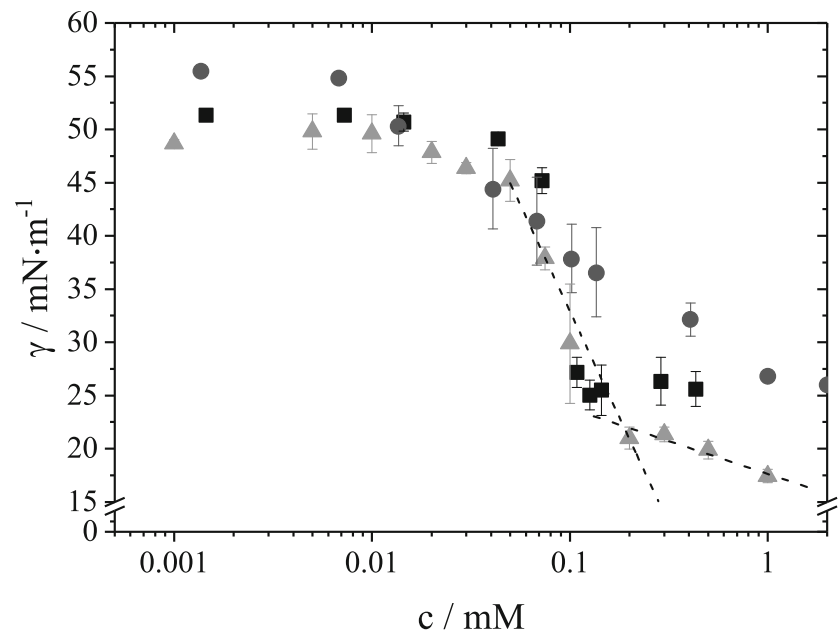

Fig. 6 Interfacial tensions $\gamma$ of the PLs DMPC, DPPC, and DSPC depending on the concentration $c$ and measured after the previously determined equilibration time for each PL ( $24 \mathrm{~h}$ for DMPC, $48 \mathrm{~h}$ for DPPC, and $168 \mathrm{~h}$ for DSPC). The dashed line that implies the slope to determine the Gibbsian isotherm, $\Gamma$, here shown exemplarily for DMPC. The intersection of both dashed lines, calculated by linear regression, is

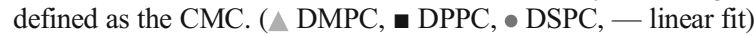

All PLs show constant interfacial tensions in the range of 49 and $55 \mathrm{mN} / \mathrm{m}$ for DMPC and DSPC, respectively, at concentrations lower than $0.01 \mathrm{mM}$. These values match the interfacial tension between PFPH and pure water $(52 \mathrm{mN} / \mathrm{m})$ implying that PLs do not influence the tensions remarkably in that regime. However, interfacial tension differs in the low concentration range, depending on the PL species. With an increasing concentration, the interfacial tension converges towards a uniform tension around $c \approx 0.01 \mathrm{mM}$. The scatter of data between 3 independent measurements, expressed by error bars of the mean, in the intermediate concentration range correlates with a two-phase coexistent region between approx. 40 and $27 \mathrm{mN} / \mathrm{m}$. These phase transitions are described in detail by Vranceanu et al. and visualized by Worthman et al. and Stottrup and Keller [34-36]. Outside the coexistence of a fluid and a solid phase, only a single phase (fluid for $>40 \mathrm{mN} / \mathrm{m}$ and solid for $<27 \mathrm{mN} / \mathrm{m}$ ) is formed which correlates with very small error bars, mostly smaller than the size of the respective symbol of the mean value. We therefore conclude that scatter of data between independent measurements is not due to the measurement technique but stems from the lack in
Table 1 Gradients of interfacial tension $-\Delta \gamma / \Delta \mathrm{t}$ at 48 and $168 \mathrm{~h}$ extrapolated equilibration interfacial tension $\gamma(t \rightarrow \infty)$ and the average standard deviation $\bar{s}_{P L}$ of the measured interfacial tensions

\begin{tabular}{lllll}
\hline $\begin{array}{l}\text { Phospholipid, } \\
\text { concentration }\end{array}$ & $\begin{array}{l}-\Delta \gamma / \Delta \mathrm{t} \text { after } 48 \mathrm{~h} \\
{[\mathrm{mN} / \mathrm{m}]}\end{array}$ & $\begin{array}{l}-\Delta \gamma / \Delta \mathrm{t} \text { after } 168 \mathrm{~h} \\
{[\mathrm{mN} / \mathrm{m}]}\end{array}$ & $\begin{array}{l}\gamma(t \rightarrow \infty) \\
{[\mathrm{mN} / \mathrm{m}]}\end{array}$ & $\begin{array}{l}\bar{s}_{P L}[\mathrm{mN} / \\
\mathrm{m}]\end{array}$ \\
\hline $\begin{array}{l}c=0.1 \mathrm{mM} \\
\text { DMPC }\end{array}$ & & & & \\
DPPC & 0.011 & 0.005 & 24.94 & 1.52 \\
DSPC & 0.220 & 0.020 & 25.56 & 1.45 \\
$c=0.14 \mathrm{mM}$ & 0.047 & & 38.47 & 3.56 \\
DPPC & & 0.000 & & \\
DSPC & 0.033 & 0.011 & 25.32 & 2.35 \\
\hline
\end{tabular}


Table 2 Critical micelle concentration $(\mathrm{CMC})$ and minimum interfacial tension $\gamma_{\min } \pm \mathrm{s}$ with $n=3$ of the examined PLs and measured with the Du Noüy ring method

\begin{tabular}{lll}
\hline Phospholipids & CMC $[\mathrm{mM}]$ & $\gamma_{\min } \pm \mathrm{s}[\mathrm{mN} / \mathrm{m}]$ \\
\hline DMPC & 0.185 & $17.42 \pm 0.62$ \\
DPPC & 0.136 & $25.03 \pm 1.40$ \\
DSPC & 0.998 & $26.03 \pm 0$ \\
\hline
\end{tabular}

reproducibility of the sample preparation in the two-phase region. This is caused by a varying of the microstructure of the liquid and solid phases in the two-phase region. Unexpectedly, the decrease of interfacial tension with increasing $c$ appears abruptly for DMPC and DPPC leading to a steep slope and, thus, a possible inaccuracy of the calculated CMC (cf. Table 2). The calculation of the $\mathrm{CMC}$ is based on the data points in the range of the linear slope. Lowest interfacial tension measured for DPPC is $25.03 \mathrm{mN} / \mathrm{m}$ at a concentration of $0.12 \mathrm{mM}$. DMPC decreases the interfacial tension even further $(17.42 \mathrm{mN} / \mathrm{m}$ at a concentration of $1 \mathrm{mM}$ ).

In contrast, for DSPC, the slope is less pronounced. Lowest determined interfacial tension at a concentration of $2 \mathrm{mM}$ is $26.03 \mathrm{mN} / \mathrm{m}$. Steric repulsion of the hydrocarbon chains is likely to be the cause for the difference in interfacial tension as has been observed for the equilibration time of the PLs. Additionally, longer chain lengths obviously lead to higher equilibrium interfacial tensions which is likely to be due to a decreasing interfacial penetration resulting in lower interfacial concentrations. This is in accordance with what has been observed by Kabalnov et al. [19]. The authors claim that interfacial tensions decrease with increasing chain lengths, but at the same time, chain lengths longer than $\left(\mathrm{C}_{13}\right)_{2} \mathrm{PC}$ do not continue the trend found for smaller chain lengths. Equilibrium experiments for chain lengths longer than $\left(\mathrm{C}_{13}\right)_{2} \mathrm{PC}$ have not been successfully performed by Kabalnov et al., and thus, equilibrium interfacial tensions have not been determined so far. Our results show that interfacial tensions can be measured even with longer fatty acid chain lengths than $\mathrm{C}_{13}$, and equilibration is possible but takes a lot of time. To gain these results, measurements took approximately $0.6 \times 10^{6} \mathrm{~s}$ (=7 days), which is much longer than what Kabalnov et al. used for their experimental set-up [19]. Additionally, measurements were performed at $20^{\circ} \mathrm{C}$, which is much lower than the transition temperature of the examined PL. This may have an influence on interfacial tensions as well and should be considered in subsequent experiments [38].

\section{Conclusions}

Our investigation of the Du Noüy ring method for the measurement of interfacial tensions between an aqueous suspension containing the phospholipid (PL) DPPC and the fluorocarbon perfluoroperhydrophenanthrene (PFPH) focused on its validity and possible difficulties or artefacts. These may arise due to the physical contact of the Du Noüy ring with the interface and its disturbance of the phospholipid monolayer as well as the necessary relaxation phase to enable measurements under equilibrium conditions.

The results show that the measured system needs a certain time to regenerate from the physical disturbance. However, the ring has only a short-term effect but neither medium nor long-term effect on the interface and does not disturb the packing of PL irreversibly. Additionally, the measurements were successfully compared with the spinning drop tensiometer as a non-invasive, yet time-consuming technique. The $\mathrm{Du}$ Noüy ring is, especially in the context of unknown systems, a very powerful method to easily obtain larger sets due to parallel sample preparation and quick measurement process and derive further parameters for the system's characterization. That applies in particular for PFPH and water that are impossible to measure with up-to-date techniques such as PAT because of identical refractive indexes.

The method was then applied to the fluorocarbon/water interface containing PL with different chain lengths but same head groups (DMPC and DSPC). The results indicate that longer chain lengths $\left(\mathrm{C}_{18} \mathrm{PC}\right)$ need more time $(168 \mathrm{~h})$ to adsorb at the interface which is likely due to increasing sterical obstruction and hydrophobic interactions with increasing chain lengths. Similar observations appear in the measurement of interfacial tensions. DMPC with shortest chain length $\left(\mathrm{C}_{14}\right.$ PC) studied here leads to the lowest interfacial tension of $17.42 \mathrm{mN} / \mathrm{m}$. For the first time, measurements between an aqueous PL suspension and a fluorocarbon, PFPH, were performed. The knowledge gained here allows to apply it to pharmaceutical emulsions and adjust the amount of PL to optimize the emulsion's properties. Future interest concerns the temperature dependency as these experiments were only performed at $20^{\circ} \mathrm{C}$, and temperature is known to influence the characteristics of PL and, thus, interfacial tensions.

Funding information Open Access funding provided by Projekt DEAL. The research leading to these results has received funding from the Phospholipid Research Center (Heidelberg, Germany). We kindly thank Lipoid (Ludwigshafen) for donating lipids to this research and Prof. Dr. Sabine Enders and Dr. Ing. Andreas Danzer from the Institute of Technical Thermodynamics and Refrigeration, KIT, for the use of their Spinning Drop Tensiometer.

\section{Compliance with ethical standards}

Conflict of interest The authors declare that they have no conflict of interest.

Open Access This article is licensed under a Creative Commons Attribution 4.0 International License, which permits use, sharing, adaptation, distribution and reproduction in any medium or format, as 
long as you give appropriate credit to the original author(s) and the source, provide a link to the Creative Commons licence, and indicate if changes were made. The images or other third party material in this article are included in the article's Creative Commons licence, unless indicated otherwise in a credit line to the material. If material is not included in the article's Creative Commons licence and your intended use is not permitted by statutory regulation or exceeds the permitted use, you will need to obtain permission directly from the copyright holder. To view a copy of this licence, visit http://creativecommons.org/licenses/by/4.0/.

\section{References}

1. van Hoogevest P, Wendel A (2014) The use of natural and synthetic phospholipids as pharmaceutical excipients. Eur J Lipid Sci Technol 116(9):1088-1107. https://doi.org/10.1002/ejlt.201400219

2. Sommerling J-H, de Matos MBC, Hildebrandt E et al (2018) Instability mechanisms of water-in-oil nanoemulsions with phospholipids: temporal and morphological structures. Langmuir 34(2): 572-584. https://doi.org/10.1021/acs.langmuir.7b02852

3. Li J, Miller R, Möhwald H (1996) Characterization of phospholipid layers at liquid interfaces. 1. Dynamics of adsorption of phospholipids at the chloroform/water interface. Colloids Surf A Physicochem Eng Asp 114:113-121. https://doi.org/10.1016/ 0927-7757(96)03521-2

4. Lee S, Kim DH, Needham D (2001) Equilibrium and dynamic interfacial tension measurements at microscopic interfaces using a micropipet technique. 2. Dynamics of phospholipid monolayer formation and equilibrium tensions at the water-air Interface. Langmuir 17(18):5544-5550. https://doi.org/10.1021/la0103261

5. Goebel A, Lunkenheimer K (1997) Interfacial tension of the water/ n -alkane Interface. Langmuir 13(2):369-372. https://doi.org/10. 1021/la960800g

6. Ushikubo FY, Cunha RL (2014) Stability mechanisms of liquid water-in-oil emulsions. Food Hydrocoll 34:145-153. https://doi. org/10.1016/j.foodhyd.2012.11.016

7. Scartazzini R, Luisi PL (1988) Organogels from lecithins. J Phys Chem 92(3):829-833

8. Sadtler VM, Krafft MP, Riess JG (1996) Achieving stable, reverse water-in-fluorocarbon emulsions. Angew Chem Int Ed Eng 35(17): 1976-1978. https://doi.org/10.1002/anie.199619761

9. Krafft MP, Giulieri F, Fontaine P et al (2001) Reversible stepwise formation of mono- and bilayers of a fluorocarbon/hydrocarbon diblock on top of a phospholipid Langmuir monolayer. A case of vertical phase separation. Langmuir 17(21):6577-6584. https://doi. org/10.1021/la010587a

10. Banks RE, Smart BE, Tatlow JC (1994) Organofluorine chemistry: principles and commercial applications. Plenum Press, New York

11. Temme S, Grapentin C, Quast C et al (2015) Noninvasive imaging of early venous thrombosis by $19 \mathrm{~F}$ magnetic resonance imaging with targeted perfluorocarbon nanoemulsions. Circulation 131: $1405-1414$

12. Wu L, Wen $X$, Wang $X$, Wang $C$, Sun $X$, Wang $K$, Zhang $H$, Williams T, Stacy AJ, Chen J, Schmieder AH, Lanza GM, Shen B (2018) Local intratracheal delivery of perfluorocarbon nanoparticles to lung cancer demonstrated with magnetic resonance multimodal imaging. Theranostics 8(2):563-574. https://doi.org/10. 7150/thno.21466

13. Cimorelli M, Angel B, Fafarman A et al (2018) Introducing a nested phase change agent with an acoustic response that depends on electric field: a candidate for myocardial perfusion imaging and drug delivery. Appl Acoust 138:9-17. https://doi.org/10.1016/j. apacoust.2018.03.028

14. Chattaraj R, Goldscheitter GM, Yildirim A, Goodwin AP (2016) Phase behavior of mixed lipid monolayers on perfluorocarbon nanoemulsions and its effect on acoustic contrast. RSC Adv 6(112):111318-111325. https://doi.org/10.1039/C6RA20328K

15. Zhang Z, Shen W, Ling J, Yan Y, Hu J, Cheng Y (2018) The fluorination effect of fluoroamphiphiles in cytosolic protein delivery. Nat Commun 9(1):1377. https://doi.org/10.1038/s41467-01803779-8

16. Lamichhane N, Dewkar KG, Sundaresan G et al (2017) [18F]-fluorinated carboplatin and [111In]-liposome for image-guided drug delivery. Int J Mol Sci 18(5):1422-0067. https://doi.org/10.3390/ ijms 18051079

17. Sun Y, Yu M, Liang S, Zhang Y, Li C, Mou T, Yang W, Zhang X, Li B, Huang C, Li F (2011) Fluorine-18 labeled rare-earth nanoparticles for positron emission tomography (PET) imaging of sentinel lymph node. Biomaterials 32(11):2999-3007. https://doi.org/10. 1016/j.biomaterials.2011.01.011

18. Riess JG (1994) Highly fluorinated systems for oxygen transport, diagnosis and drug delivery. Colloids Surf A Physicochem Eng Asp 84(1):33-48. https://doi.org/10.1016/0927-7757(93)02696-C

19. Kabalnov A, Weers J, Arlauskas R et al (1995) Phospholipids as emulsion stabilizers. 1. Interfacial tensions. Langmuir 11(8):29662974. https://doi.org/10.1021/la00008a020

20. Li J, Miller R, Möhwald H (1996) Characterisation of phospholipid layers at liquid interfaces 2. Comparison of isotherms of insoluble and soluble films of phospholipids at different fluid/water interfaces. Colloids Surf A Physicochem Eng Asp 114:123-130. https://doi.org/10.1016/0927-7757(96)03522-4

21. Hildebrandt E, Dessy A, Sommerling J-H, Guthausen G, Nirschl H, Leneweit $G$ (2016) Interactions between phospholipids and organic phases: insights into lipoproteins and Nanoemulsions. Langmuir 32(23):5821-5829. https://doi.org/10.1021/acs.langmuir.6b00978

22. Hildebrandt E, Sommerling J-H, Guthausen G et al (2016) Phospholipid adsorption at oil in water versus water in oil interfaces: implications for interfacial densities and bulk solubilities. Colloids Surf A Physicochem Eng Asp 505:56-63. https://doi. org/10.1016/j.colsurfa.2015.12.024

23. Lecomte Du Noüy P (1919) A new apparatus for measuring surface tension. J Gen Physiol:521-524

24. Lunkenheimer K, Wantke K-D (1981) Determination of the surface tension of surfactant solutions applying the method of Lecomte du Noüy (ring tensiometer). Colloid Polym Sci 259(3):354-366. https://doi.org/10.1007/BF01524716

25. Harkins WD, Jordan HF (1930) A method for the determination of surface and interfacial tension from the maximum pull on a ring. $\mathrm{J}$ Am Chem Soc 52:1751-1772

26. Drelich J, Fang C, White CL (2002) Measurements of interfacial tensions in fluid-fluid systems. Encylclopedia of Surface and Colloid Science:3152-3166

27. Kovach I, Koetz J, Friberg SE (2014) Janus emulsions stabilized by phospholipids. Colloids Surf A Physicochem Eng Asp 441:66-71. https://doi.org/10.1016/j.colsurfa.2013.08.065

28. Vonnegut B (1942) Rotating bubble method for the determination of surface and interfacial tensions. Rev Sci Instrum 13(1):6-9. https://doi.org/10.1063/1.1769937

29. Benmekhbi M, Simon S, Sjöblom J (2014) Dynamic and rheological properties of span 80 at liquid-liquid interfaces. J Dispers Sci Technol 35(6):765-776. https://doi.org/10.1080/01932691.2013. 811573

30. Arajo Lopes SCd, Santos Giuberti Cd, Ribeiro TG et al. (2013) Liposomes as carriers of anticancer drugs. In: Rangel L (ed) 
Cancer Treatment - Conventional and Innovative Approaches. InTech, 85-124

31. Fiske CH, Subbarow Y (1925) The colorimetric determination of phosphorus. J Biol Chem 66:375-400

32. Vargaftik NB, Volkov BN, Voljak LD (1983) International tables of the surface tension of water. J Phys Chem Ref Data 12(3):817-820. https://doi.org/10.1063/1.555688

33. Hildebrandt E, Nirschl H, Kok RJ, Leneweit G (2018) Adsorption of phospholipids at oil/water interfaces during emulsification is controlled by stress relaxation and diffusion. Soft Matter 14(19): 3730-3737. https://doi.org/10.1039/c8sm00005k

34. Vrânceanu M, Winkler K, Nirschl H, Leneweit G (2008) Surface rheology and phase transitions of monolayers of phospholipid/ cholesterol mixtures. Biophys J 94(10):3924-3934. https://doi. org/10.1529/biophysj.107.104851

35. Worthmann LA, Nag K, Davis PJ et al (1997) Cholesterol in condensed and fluid phosphatidylcholine monolayers studied by epifluorescence microscopy. Biophys J 72(6):2569-2580. https:// doi.org/10.1016/S0006-3495(97)78900-8

36. Stottrup BL, Keller SL (2006) Phase behavior of lipid monolayers containing DPPC and cholesterol analogs. Biophys J 90(9):31763183. https://doi.org/10.1529/biophysj.105.072959

37. Israelachvili JN, Mitchell DJ (1975) A model for the packing of lipids in bilayer membranes. Biochim Biophys Acta Biomembr 389(1):13-19. https://doi.org/10.1016/0005-2736(75)90381-8

38. Purdon AD, Tinker DO, Neumann AW (1980) The temperature dependence of surface tension and critical micelle concentration of egg lysolecithin. Colloid Polym Sci 1980(258):1062-1069

Publisher's note Springer Nature remains neutral with regard to jurisdictional claims in published maps and institutional affiliations. 